\title{
Human Heart Pulse Wave Responses Measured Simultaneously at Several Sensor Placements by Two MR-Compatible Fibre Optic Methods
}

\author{
Teemu Myllylä,, ${ }^{1}$ Vesa Korhonen, ${ }^{2}$ Erkki Vihriälä, ${ }^{1}$ Hannu Sorvoja, ${ }^{1}$ Tuija Hiltunen, ${ }^{2}$ \\ Osmo Tervonen, ${ }^{2}$ and Vesa Kiviniemi ${ }^{2}$ \\ ${ }^{1}$ Optoelectronics and Measurement Techniques Laboratory, University of Oulu, Oulu, Finland \\ ${ }^{2}$ Department of Diagnostic Radiology, Oulu University Hospital, Oulu, Finland \\ Correspondence should be addressed to Teemu Myllylä, teemu.myllyla@ee.oulu.fi
}

Received 20 July 2012; Accepted 15 November 2012

Academic Editor: Rongping Wang

Copyright (๑) 2012 Teemu Myllylä et al. This is an open access article distributed under the Creative Commons Attribution License, which permits unrestricted use, distribution, and reproduction in any medium, provided the original work is properly cited.

This paper presents experimental measurements conducted using two noninvasive fibre optic methods for detecting heart pulse waves in the human body. Both methods can be used in conjunction with magnetic resonance imaging (MRI). For comparison, the paper also performs an MRI-compatible electrocardiogram (ECG) measurement. By the simultaneous use of different measurement methods, the propagation of pressure waves generated by each heart pulse can be sensed extensively in different areas of the human body and at different depths, for example, on the chest and forehead and at the fingertip. An accurate determination of a pulse wave allows calculating the pulse transit time (PTT) of a particular heart pulse in different parts of the human body. This result can then be used to estimate the pulse wave velocity of blood flow in different places. Both measurement methods are realized using magnetic resonance-compatible fibres, which makes the methods applicable to the MRI environment. One of the developed sensors is an extraordinary accelerometer sensor, while the other one is a more common sensor based on photoplethysmography. All measurements, involving several test patients, were performed both inside and outside an MRI room. Measurements inside the MRI room were conducted using a 3-Tesla strength closed MRI scanner in the Department of Diagnostic Radiology at the Oulu University Hospital.

\section{Introduction}

Optical measurements in the magnetic resonance imaging (MRI) environment commonly employ MR-compatible fibres. This is because the primary light sources and detectors used in these measurements cannot be placed inside an MRI room due to interference problems $[1,2]$. Photoplethysmogram (PPG) measurements are generally conducted using an $\mathrm{LED} /$ photo detector pair placed on the skin near each other. To utilize this type of measurement in the MRI environment, light generated by an LED must be guided inside the MRI room by an MR-compatible fibre whose other end is attached on the patient's skin [3-5]. Light reflected back from tissue is then guided by a receiver fibre to a photo detector placed outside the MRI room. The attenuation effect caused by such long fibres can be compensated for by the use of a very sensitive photodetector and a high-power LED. In this way, pulsations in blood flow can be noninvasively monitored in the MRI environment in all parts of the human body, as long as the illuminating light beam can reach sufficiently deep within tissue and the detector is sensitive enough to record fluctuations in the intensity of the received light.

1.1. Propagation of Light in Human Tissue. Measurement of light that travels in tissue is often referred to as diffusive optical imaging (DOI) [6]. As light traverses a tissue, movements within the tissue produce absorption and scattering changes, which the detector records as intensity changes. These movements arise mainly as a consequence of blood flow related to heart pumping. The distance between the light source and the detector determines how deep within the tissue blood flow pulsations can be detected [7]. When light 
illuminates a tissue, it begins to scatter and diffuse, producing a banana-shaped light diffusion path $[8,9]$. As a result, detectors closer to the light source detect superficial portions of the tissue, while detectors placed further from the source are capable of deeper interrogation. Thus, by increasing the distance between the light source and the detector, we may measure blood flow pulsations deeper within the body.

As the distance increases, diffusion allows less photons to be detected, resulting in a poor signal-to-noise ratio. However, this can be improved by using different modulation techniques [10]. Sophisticated measurement methods even allow sensing pulsations in the grey matter of the brain [6], provided that the distance between the source and the detector is sufficiently wide. Simulations show that this distance needs to be more than $2.5 \mathrm{~cm}$ when using red and infrared light [10]. Additionally, near-infrared spectroscopy (NIRS), a method related to DOI, is used in brain activity studies, as it allows using several wavelengths or a wider frequency band of light to illuminate tissue. When light traverses a tissue, each wavelength provides a specific response. By simultaneously measuring responses at several wavelengths, proportions of certain elements within the tissue, such as oxygen level, can be observed $[11,12]$.

1.2. Heart Rate Detection by Accelerometers. In addition to measurements based on DOI, we use an MR-compatible fibre optic measurement method, based on accelerometer sensors, to determine heart rate on the basis of skin movements caused by heart pumping. These accelerometer sensors enable measuring the velocity of pulse waves in arterial blood during fMRI within our noninvasive blood pressure device [13]. This value can then be used to estimate diastolic blood pressure.

1.3. Simultaneous Use of Functional Magnetic Resonance Imaging and Blood Flow Pulsation Measurement. The simultaneous use of functional magnetic resonance imaging (fMRI) and measurement methods described in this paper offer great possibilities for the study of neural activity. Since fMRI provides accurate spatial information and DOI-based measurement accurate time-domain information [14], these methods complement each other.

Furthermore, blood pressure pulsations, measured by PPG and accelerometer sensors, are commonly utilized both in clinical applications and in biomedical research. The main purpose of this paper is to show that these pulsations can also be measured during MRI. Moreover, new ideas may also emerge from the ability to utilize the MRI environment. As an example, we additionally present PTT results for the measured signals to show that the responses of the signals are accurate and sufficiently clear for the analysis of biosignals.

\section{Method of Measurements}

Applied methods are used to determine blood pressure pulsations from the human body. Our NIRS measurement device utilizes lock-in amplification, which gives a high signal-to-noise ratio and enables wavelength encoding, allowing different wavelengths to be distinguished from one another. Up to four different colours of LEDs can be applied to simultaneously illuminate one spot. Light from these LEDs is collected by a fibre bundle into a single-ended fibre, which illuminates the tissue as a one-point source. Light reflected back from the illuminated tissue is measured by a fibre detector, and the colours are distinguished from each other by a demodulator. These two fibre sensors and measurement methods allow the measurement of such parameters as heart pulse shape, pulse transit time (PTT), and blood oxygen saturation. Furthermore, the results can be combined with fMRI measurement results, since they are being conducted simultaneously with fMRI. Possible correlations between these signals may provide significant information for brain activity studies.

2.1. Sensor Arrangements. Figure 1 shows all sensor placements used in the measurements, which can all be conducted simultaneously both outside and inside the MRI room. Using standard methods, signals received from the sensors are first amplified and filtered, before the received analog data is collected on a National Instruments data acquisition card and processed using a LabVIEW program. For accelerometer sensors, the sampling rate was $10 \mathrm{kHz}$ and for NIRS/PPG sensors $40 \mathrm{kHz}$. For electrocardiogram (ECG) sensors, used outside the MRI room, the sampling rate was $40 \mathrm{kHz}$ and for electroencephalography (EEG)/ECG sensors, used inside the MRI room, the rate was $5 \mathrm{kHz}$. Measurements inside the MRI operating room were performed by a 3-Tesla strength closed MRI scanner in the Department of Diagnostic Radiology at the Oulu University Hospital.

Presented in the following paragraphs are examples of the five measurement arrangements conducted during this study.

Measurement 1. This first measurement, conducted in the Optoelectronics and Measurement Techniques Laboratory, was mainly based on the PPG method. One infrared LED $(830 \mathrm{~nm})$ was placed in the middle of the test subject's forehead with a fibre source-detector distance of $2.9 \mathrm{~cm}$. Another infrared LED $(905 \mathrm{~nm})$ was placed in the big toe of the left foot with a fibre source-detector distance of $2 \mathrm{~mm}$. A third LED was red $(660 \mathrm{~nm})$, and it was placed on the forefinger of the subject's right hand. The fibre source-detector distance was $1.3 \mathrm{~cm}$. In all these situations, $10 \mathrm{~m}$ long MRI-compatible fibre bundles were used for the LED source and photo detector/receiver. Additionally, since FINAPRES can be regarded as a reliable reference device for the measurement [15], a Finapres Ohmeda 2300 sensor was placed in the left-hand forefinger to obtain a reference signal for a comparison to be made with signals from our fibre optic sensors. Three ECG sensors were placed as described in Figure 1. While the measurements were in progress, the subject, a 29-year-old male, was sitting still.

Measurement 2. Also the second measurement was carried out in the Optoelectronics and Measurement Techniques Laboratory and relied mainly on the PPG method. However, it only employed one wavelength $(830 \mathrm{~nm})$. In this measurement, PPG sensors were placed in the middle of the subject's 


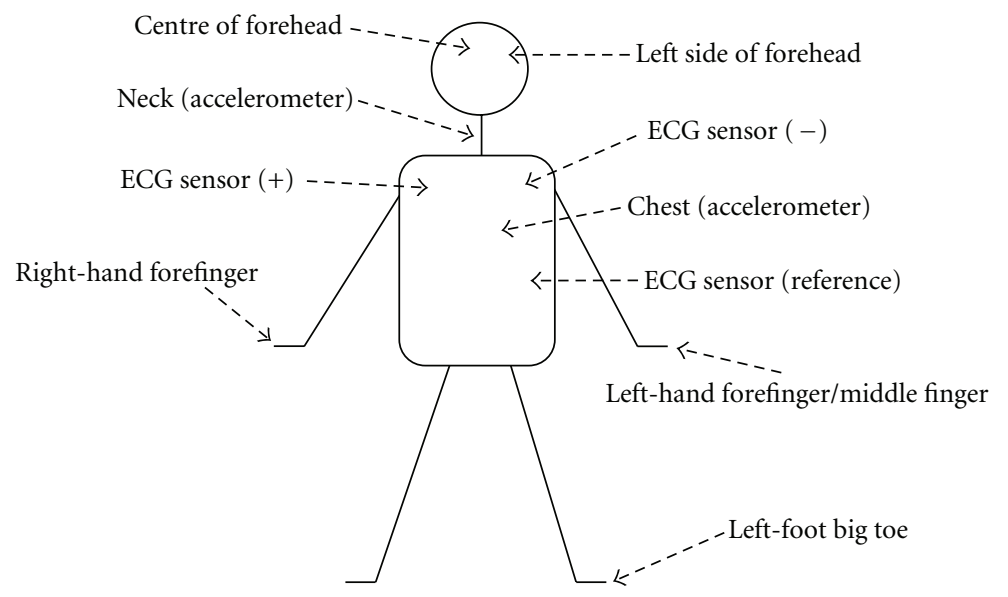

Figure 1: Sensor placements used in the measurements. Three ECG sensors were placed as follows: One on the left shoulder (minus), one on the right shoulder (plus), and a third one on the stomach, to left of the navel. PPG-based sensors, on the other hand, were placed in one finger on both hands, on the forehead, and on a toe. In these measurements, source-detector distances varied from $2 \mathrm{~mm}$ to $30 \mathrm{~mm}$. Finally, accelerometer sensors were placed on the chest and in the neck, on the carotid artery. All optical fibres used in the measurements were $10 \mathrm{~m}$ long and MR compatible.

forehead, on the left side of the forehead, and in the lefthand forefinger. Transmitter-receiver distances were $2.9 \mathrm{~cm}$ in the middle of the forehead, $2.8 \mathrm{~cm}$ on the left side of the forehead, and $1.3 \mathrm{~cm}$ in the forefinger. The used PPG sensor, located in the right-hand forefinger, was the same as in Measurement 1. Also the ECG and accelerometer sensors were placed as in Measurement 1 . The test subject was a 41year-old male.

Measurement 3. The third measurement only employed the PPG method. One fibre source and three fibre receivers were used, placed side by side, with the fibre source followed by the receivers. The distances between the receivers and the transmitter were 1,2 , and $3 \mathrm{~cm}$. All sensors were placed at the centre of the forehead. In addition, a single PPG sensor was placed on the right-hand forefinger. This measurement was also performed in the Optoelectronics and Measurement Techniques Laboratory, and the test subject was the same man as in Measurement 1.

Measurement 4. In the fourth measurement, a PPG sensor, placed in the middle of the test subject's forehead, was used at two wavelengths, $830 \mathrm{~nm}$ and $905 \mathrm{~nm}$. In addition, accelerometer sensors were located both on the chest and on the carotid artery in the neck. The distance between the chest and neck sensors was $15 \mathrm{~cm}$. Conducted on a 20 -yearold female test subject, this measurement was done inside the MRI operating room of the Department of Diagnostic Radiology at the Oulu University Hospital.

Measurement 5. The arrangement of the fifth measurement was otherwise identical to that of Measurement 4, but it involved wearing an EEG cap and employed three wavelengths ( $660 \mathrm{~nm}, 830 \mathrm{~nm}$, and $905 \mathrm{~nm}$ ) instead of two. A PPG sensor was placed in the middle of the subject's forehead with a fibre source-detector distance of $3.0 \mathrm{~cm}$. Accelerometer sensors were placed on the chest and on the carotid artery in the neck at a distance of $23 \mathrm{~cm}$. ECG was measured by Brain Product's ready-to-use EEG/fMRI system, which includes an EEG cap and an amplifier. The test subject wore an electrode cap connected to a shielded amplifier located inside the MRI chamber, near the subject's head. From the electrodes of the cap, digitized signals were sent to a control room via a fiber optic cable. Each ECG was acquired at the maximum sampling rate of the amplifier $(5 \mathrm{kHz})$, and the used bandwidth was DC-250 Hz. Conducted on a 26-yearold male test subject, this measurement was conducted inside the MRI operating room of the Department of Diagnostic Radiology at the Oulu University Hospital.

2.2. Determination of Peak Time. To determine the delay times of pulses in different sensor positions, $\mathrm{R}$ wave peaks of pulses were identified by searching for the maximum value of each time interval in synchronously measured signals. In determining these time intervals, the signal response with the clearest and most obvious peak was chosen as a reference. In most cases, the method based on the maximum value search finds the narrowest peak and ignores measurement errors caused by wide pulses, which are often a problem when measuring at long source-detector distances. However, to ensure that the right peaks were identified, the peaks were marked as dots on the signal curves and then checked visually that they are in the right places.

\section{Results}

Shown below are examples of blood flow pulsation responses measured by the methods described in the previous section. Our main interest was to record the pulse shape, particularly the minimum amplitude before each pulsation wave and the rising edge of the pulsation wave. We also calculated pulse delays between different sensor placements. 


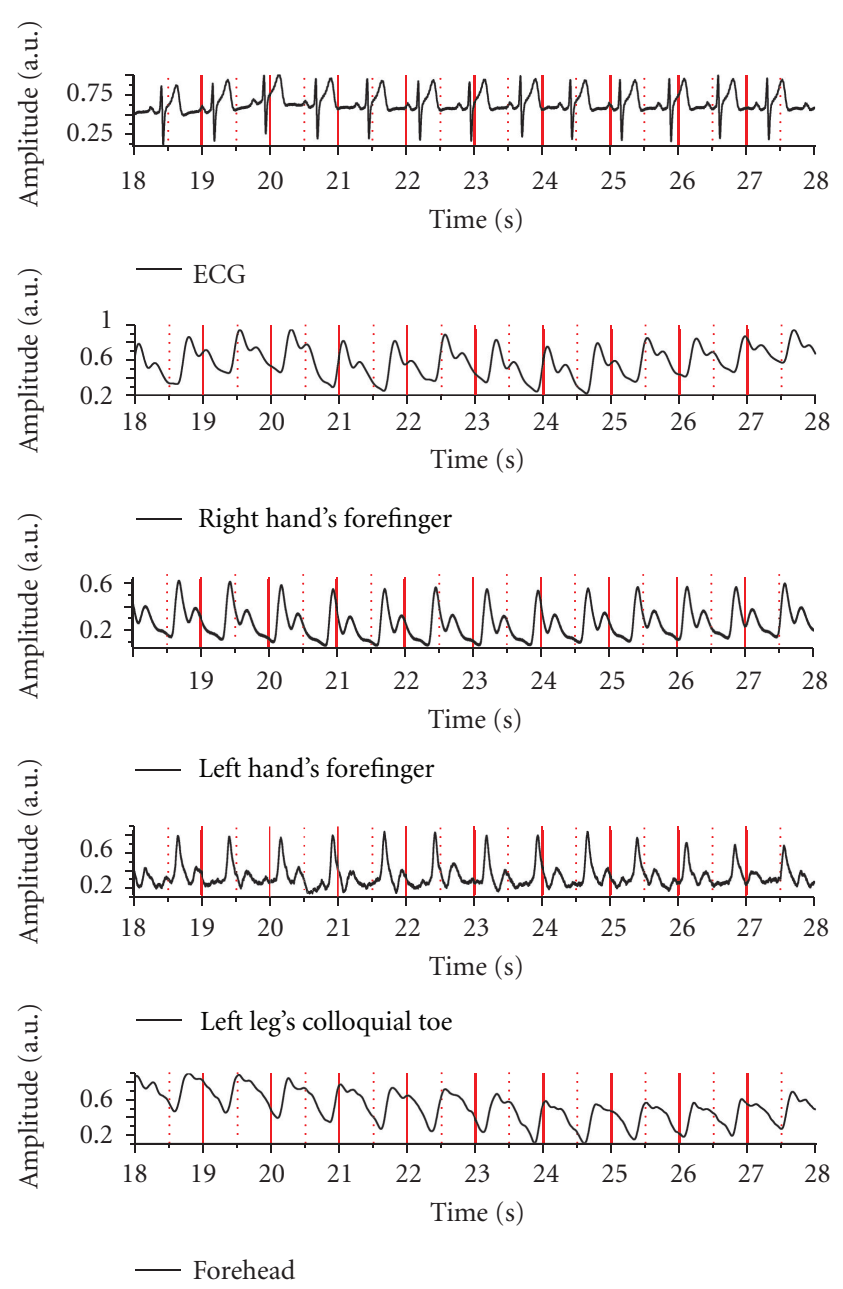

Figure 2: Measurement 1: ECG and blood flow pulsation responses in different parts of the human body.

Measurement 1. All signals presented in Figure 2 were normalized 0 to 1 . As seen from the figure, the sharpest positive peak of the ECG signal is always the first one, which makes it reasonable to compare other signals to it. All positive peaks in Figure 2 were determined from raw blood flow pulsations. Our results are shown in Table 1, with the time points matching the positive peaks presented in Figure 2 shown first. Lower down in the table are calculated time differences compared to the corresponding positive peaks in ECG signals in different phases. As indicated by the maximum standard deviation of 0.016 , shown in Table 2, these time differences remain fairly constant. Furthermore, the maximum standard deviation comes from the forehead signal, where the distance between the source and the detector of the sensor is the largest.

An underlying reason for the different signal shapes shown in Figure 2 is variance in the source-receiver distance. The shorter the distance, the sharper the shape of the signal. Figure 2 and the calculated PTT values, shown in Table 2, also reveal that the corresponding positive peaks from the right-hand and left-hand forefingers are not simultaneous.
However, for the most part, this can be explained by the position of the hands during the measurement and by the influence of gravitation. The test person was in a sitting position with the left arm lying on a table and the right arm raised approximately at head level. This was done to demonstrate the effect of the gravitation. Additionally, it must be noted that blood flow pulses measured from the foot came earlier than the equivalent pulses from the fingers, although the physical distance is shorter from the heart to the hand than to the foot.

According to Bernoulli's principle [16], gravitation causes blood to flow faster downwards than upwards. If the person is standing or sitting, as in this measurement, pulse velocity is faster in the foot than in the hand, which is resting in the horizontal plane. This explains why the time difference is smaller for a person in a supine position. Gravitation is also the reason why the positive peak in the left hand occurs earlier than in the forehead, although the distance to the forehead is shorter. But when the arm is raised (as was the case of the right hand), the pulse transit time becomes longer, approaching that measured from the forehead.

Measurement 2. All signals presented in Figure 3 were also normalized 0 to 1 . Again, the ECG signal provided the reference against which other signals were compared. The average time differences were $0.260 \mathrm{~s}$ for the right hand, $0.338 \mathrm{~s}$ for the left hand, 0.362 for the left side of the forehead, and 0.401 for the middle of the forehead. The equivalent standard deviations were 0.002 for the left hand, 0,003 for the right hand, 0.014 for the centre of the forehead, and 0.021 for the left side of the forehead. The order of arrival of the corresponding positive peaks was ECG, right hand, left hand, and head.

Also the starting point of the rising edge of each pulse was determined. Again, the reference point was the positive peak of the ECG signal. The average time differences were $0.117 \mathrm{~s}$ for the right hand, $0.162 \mathrm{~s}$ for the left hand, $0.181 \mathrm{~s}$ for the left side of the forehead, and $0.200 \mathrm{~s}$ for the middle of the forehead. The order was the same as with positive peaks, and the ratio of time differences was almost identical. Corresponding standard deviations were 0.026, 0.005, 0.020, and 0.017 , respectively.

Measurement 3. The purpose of the third measurement was to investigate pulse shape differences and pulse time delays in measurements conducted on the forehead at varying sourcedetector distances. As a reference signal, we used a single PPG sensor on a finger. As Figure 4 illustrates, the positive peak from the finger was the first to arrive in most cases. The average time differences compared to the finger were $0.032 \mathrm{~s}$ for $3 \mathrm{~cm}, 0.034 \mathrm{~s}$ for $2 \mathrm{~cm}$, and $0.058 \mathrm{~s}$ for $1 \mathrm{~cm}$. For sensors placed on the forehead, positioning of the sensor row is relevant, since the direction of blood flow affects the pulse delays measured by the detectors.

The equivalent standard deviations for the different distances were $0.038,0.024$, and 0.006 . The largest standard deviation, obtained at a source-detector distance of $3 \mathrm{~cm}$, can be explained by the shape of the signal. 
TABLE 1: Positive peak times and pulse transit times (PTT) compared to ECG.

\begin{tabular}{|c|c|c|c|c|c|c|c|c|c|c|c|c|c|}
\hline \multicolumn{14}{|c|}{ Peak times (s) } \\
\hline ECG & 18.412 & 19.165 & 19.919 & 20.679 & 21.433 & 22.183 & 22.936 & 23.683 & 24.415 & 25.144 & 25.868 & 26.585 & 27.304 \\
\hline Right hand & 18.794 & 19.543 & 20.298 & 21.062 & 21.820 & 22.558 & 23.309 & 24.061 & 24.799 & 25.528 & 26.250 & 26.965 & 27.683 \\
\hline Left hand & 18.691 & 19.440 & 20.190 & 20.951 & 21.710 & 22.460 & 23.210 & 23.960 & 24.690 & 25.420 & 26.145 & 26.865 & 27.580 \\
\hline Left leg & 18.659 & 19.415 & 20.170 & 20.93 & 21.691 & 22.434 & 23.19 & 23.942 & 24.668 & 25.397 & 26.122 & 26.834 & 27.552 \\
\hline Forehead & 18.791 & 19.528 & 20.257 & 21.02 & 21.788 & 22.561 & 23.318 & 24.034 & 24.777 & 25.514 & 26.215 & 26.916 & 27.664 \\
\hline \multicolumn{14}{|l|}{$\operatorname{PTT}(\mathrm{s})$} \\
\hline ECG & 0.000 & 0.000 & 0.000 & 0.000 & 0.000 & 0.000 & 0.000 & 0.000 & 0.000 & 0.000 & 0.000 & 0.000 & 0.000 \\
\hline Right hand & 0.382 & 0.378 & 0.379 & 0.383 & 0.387 & 0.375 & 0.373 & 0.378 & 0.384 & 0.384 & 0.382 & 0.380 & 0.379 \\
\hline Left hand & 0.279 & 0.275 & 0.271 & 0.272 & 0.277 & 0.277 & 0.274 & 0.277 & 0.275 & 0.276 & 0.277 & 0.280 & 0.276 \\
\hline Left leg & 0.247 & 0.250 & 0.251 & 0.251 & 0.258 & 0.251 & 0.254 & 0.259 & 0.253 & 0.253 & 0.254 & 0.249 & 0.248 \\
\hline Forehead & 0.379 & 0.363 & 0.338 & 0.341 & 0.355 & 0.378 & 0.382 & 0.351 & 0.362 & 0.370 & 0.347 & 0.331 & 0.360 \\
\hline
\end{tabular}

TABle 2: Average and standard deviation of PTT calculated from the values presented in Table 1 .

\begin{tabular}{lcc}
\hline PTT $(\mathrm{s})$ & Average & Standard deviation \\
\hline ECG & 0.000 & 0.000 \\
Right hand & 0.380 & 0.004 \\
Left hand & 0.276 & 0.003 \\
Left leg & 0.252 & 0.004 \\
Forehead & 0.358 & 0.016 \\
\hline
\end{tabular}

We also investigated the starting points of pulses, that is, the starting point of the rising edge of every pulse. When compared to the equivalent positive peaks, the average time differences were $0.158 \mathrm{~s}$ for $1 \mathrm{~cm}, 0.152 \mathrm{~s}$ for $2 \mathrm{~cm}, 0.130 \mathrm{~s}$ for $3 \mathrm{~cm}$, and $0.136 \mathrm{~s}$ for the finger. Corresponding standard deviations were $0.005,0.024,0.028$, and 0.031 , respectively. An explanation for the largest standard deviation, obtained from the finger, is the gentle rising edge of the finger pulse in some points. All the results shown here were calculated from the period shown in Figure 4, but the results remain similar even for longer periods.

Measurement 4. In these measurements, carried out in the MRI environment, accelerometer signals (chest and neck) were extraordinarily clear, facilitating comparison between accelerometer and PPG signal delays. No interference is noticeable, despite simultaneous MR imaging.

Obviously, signal peaks measured from the chest arrived first. Thus, signal peaks measured from the neck and the forehead were now compared to them. The average time difference between chest and neck signals was $0.053 \mathrm{~s}$ in the tensecond period shown in Figure 5. The equivalent standard deviation was 0.019 , but if two somewhat questionable peaks ( $\sim 205.8 \mathrm{~s}$ and $\sim 208.5 \mathrm{~s})$ are excluded, the standard deviation falls to 0.002 . In this case, the average time difference between the positive peak of the chest and neck signal is $0.045 \mathrm{~s}$. Positive peaks of PPG signals, measured from the forehead, clearly arrive after the corresponding peaks from the chest. The average time difference between chest signals was $0.237 \mathrm{~s}$ at $830 \mathrm{~nm}$ and $0.235 \mathrm{~s}$ at $905 \mathrm{~nm}$, and the corresponding standard deviations were 0.035 and 0.032 , respectively. These
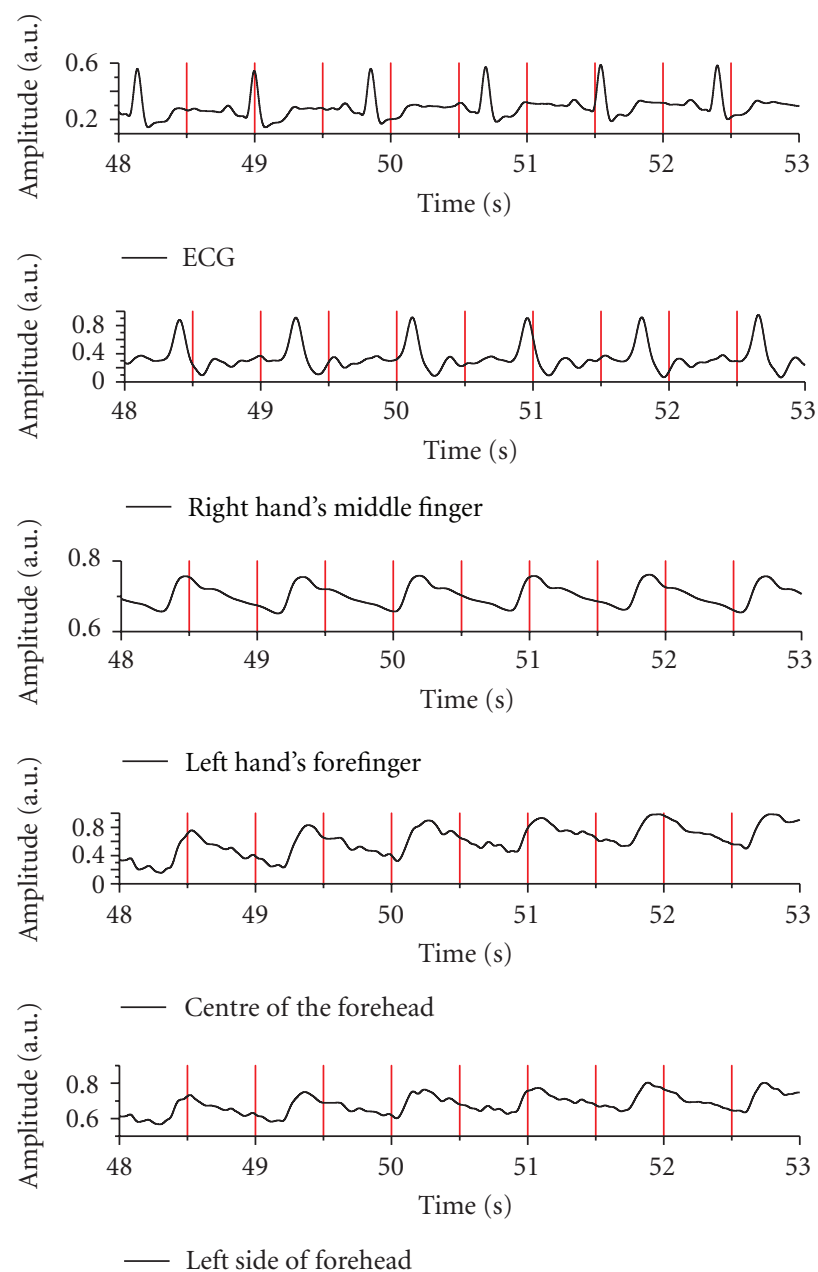

Figure 3: Measurement 2: ECG and blood flow pulsation responses in different parts of the human body.

results are very close to each other, which is to be expected, since they are measuring the same thing in exactly the same place. Only the pulse shape differs, because of different wavelengths. 

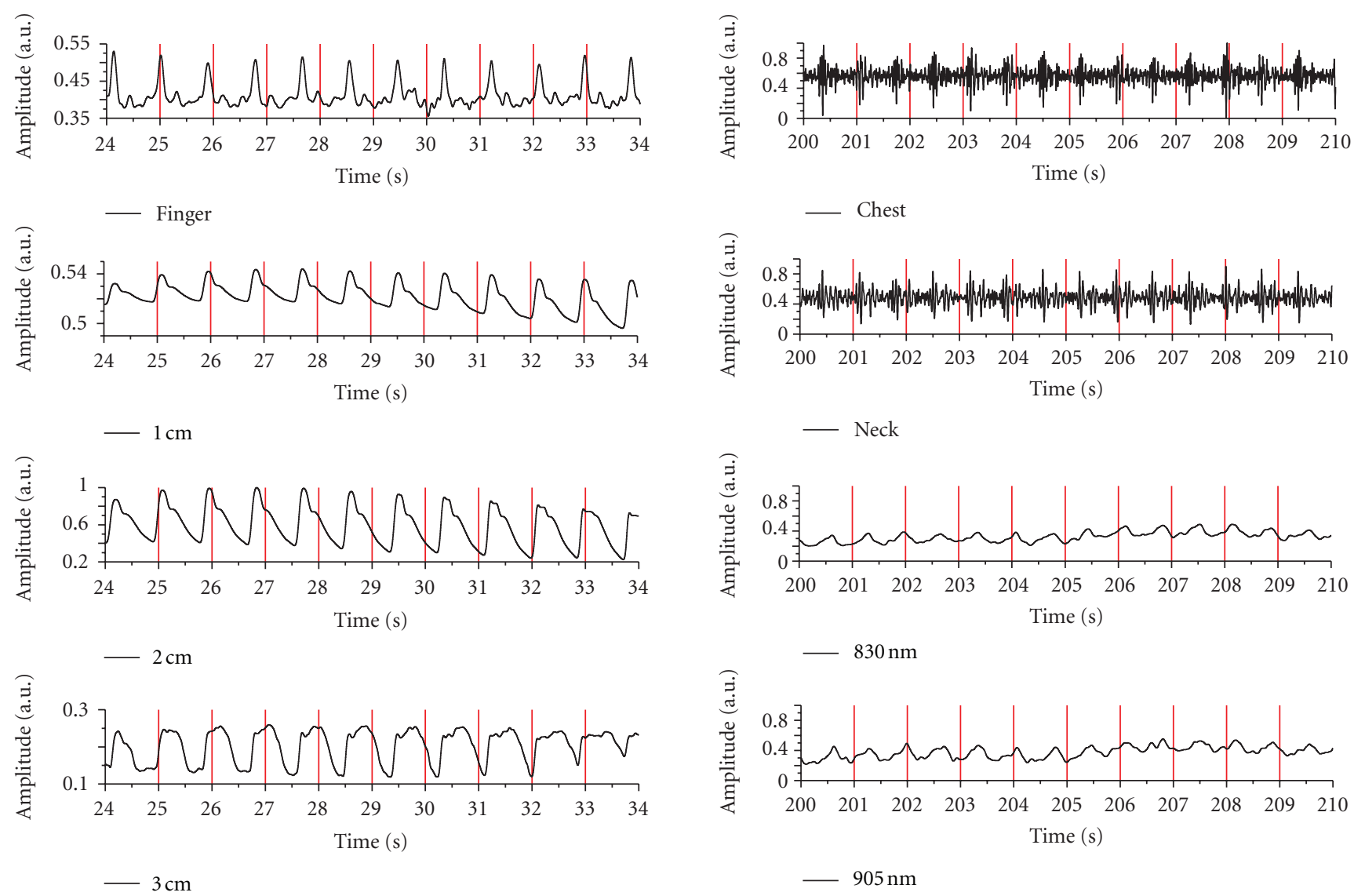

Figure 4: Measurement 3: PPG sensors placed on finger and forehead at 3 different source-detector distances.

Measurement 5. Measurement 5 explored responses of pulse waves using three different measurement methods: ECG, accelerometers, and PPG/NIRS presented in Figure 6. Compared to the equivalent positive peaks of ECG signals, the recorded time differences between positive peaks were identical to those of earlier measurements. However, the signal-to-noise ratio of accelerometer signals was weak, owing to poor sensor positioning especially on the neck.

In most cases, it is fairly easy to use an accelerometer to sense pulsations in the chest, because the sensor is placed near the heart. In the neck, however, tiny skin movements are sometimes more difficult to sense and depend greatly on the person to be measured, as evidenced by Measurement 5 . Reasons for this can be fysiological, but in most cases the arteries are located a little deeper and blood flow pulsations cause less skin movements. In this type of situation, proper placement of the sensor on the neck is crucial. The best position for a sensor can normally be found by pressing your fingers gently on the neck to feel where blood flow pulsations are most pronounced. Furthermore, the accelerometer has to be attached tightly, yet gently, to prevent skin movements caused by pressing the skin too hard. Usually it is not very difficult to achieve a sufficient signal-to-noise ratio, but sometimes this can only be accomplished after several sensor replacements.

FIgURE 5: Measurement 4: Accelerometer and PPG signals measured inside the MRI room during MRI.

\section{Discussion}

The quality of the pulse wave signals measured by the presented sensors was in all cases highly dependent on sensor positioning. Accelerometer sensors must be positioned carefully above a pulsating surface and placed tightly on the skin, but not too tightly, as that would prevent pulse wave vibrations on the skin from reaching the sensor. Likewise, for sensors based on PPG, a tight attachment of the fibre to the skin is important. A loose attachment allows the fibre to move in relation to skin, causing interfering peaks. Additionally, if the direction of the fibre output does not stay the same for the entire measurement (perpendicular to skin), either too much or too little, light can be reflected to the detector. This disturbs measurements based on very sensitive detectors and high amplification, especially with a wide source-detector distance. Also, when using long fibres, movement of the fibres may affect the signal response, if the attachment is not sufficiently secure. However, with proper sensor attachment, both sensors provide reliable data for further signal processing.

In terms of further signal analysis, accurate pulse shape measurement is highly important. These measurements are greatly affected by the wavelength of the illuminating light, measurement placement, and positioning of sensors - and in the case of PPG sensors, also by the distance between the fibre 


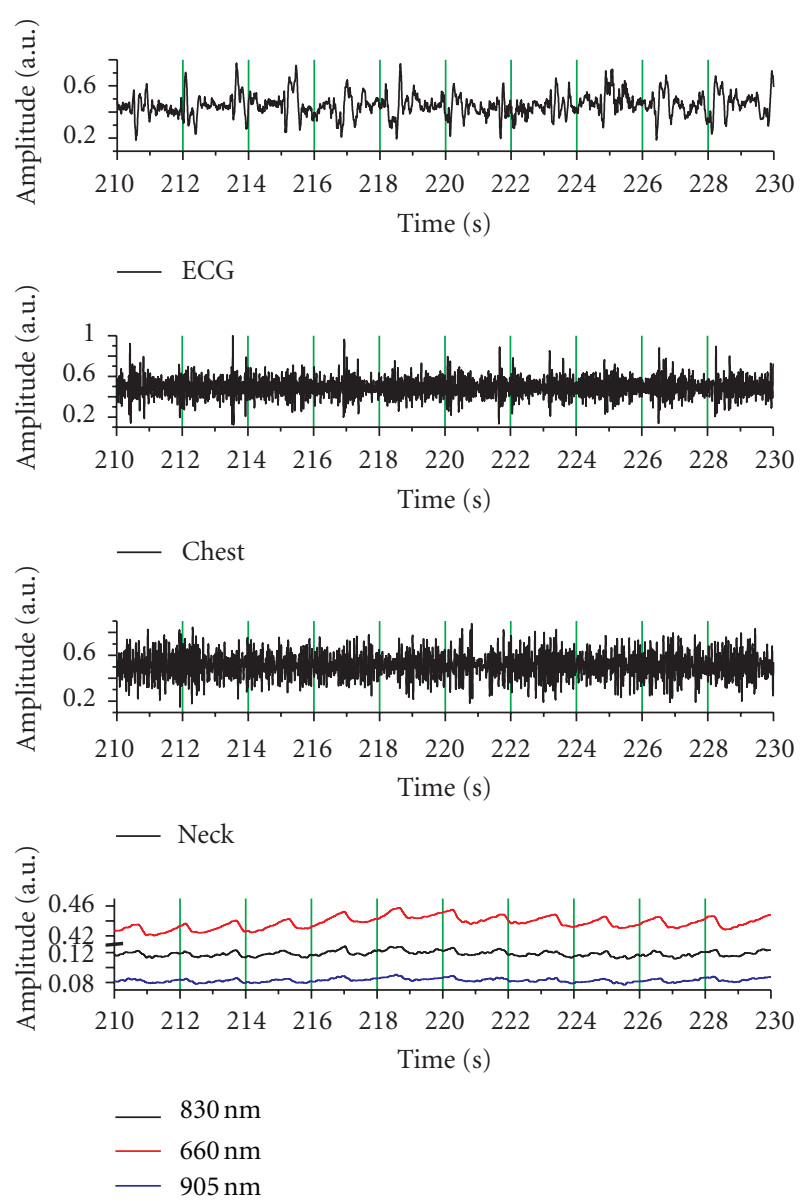

Figure 6: Measurement 5: ECG, accelerometer and PPG/NIRS signals measured inside the MRI room during MRI.

source and the fibre detector. There are also some aspects that must be considered when measuring time delays related to bloodstream pulsations at different locations. First of all, the shape of pulsation signals varies in relation to the measuring position. Therefore, for example, if measurements are based on the maximum peak values of pulses, the results will differ from those obtained from the starting point of pulses. These changes in pulse shapes are related to the filtering effect of the circulatory system [17]. One way of minimizing this effect involves measuring pulse transition times using the starting point of pulses. In the most cases, the starting point of a pulse is the minimum recorded just before the maximum peak of each time interval. However, if signal pulsation is disturbed or the riding edge of the $\mathrm{R}$ wave pulse is not clear, then the starting point has to be estimated visually.

Furthermore, if delays are determined by ECG signals, it must be noted that the location of the peak values of ECG signals changes with the location of the electrodes. This should be considered when ECG signals are used, for example, to determine PTT. Additionally, the shape of the ECG signal is not directly comparable to the shape of bloodstream pulsation signals, and ECG signals also experience an additional delay (preejection period). Therefore, when determining PTT, an accelerometer sensor, positioned on the chest above the heart, will probably provide a more reliable starting time for each heart pulse wave than ECG, assuming that the accelerometer sensor is properly positioned. Additionally, in measurements conducted on the chest, signal pulsations from the accelerometer sensor were sharper and less disturbed by breathing than those measured by the PPG sensor.

\section{Conclusions}

Our measurements demonstrate that it is possible to measure blood pressure pulsation and propagation of these waves generated by each heart pulse in different body areas during fMRI by using fibre optics. Heart rate and delays of pulsation waves between different sensor placements can be determined with an accuracy of milliseconds. Pulse shapes can also be determined with a fibre source-detector distance of $3 \mathrm{~cm}$.

When measuring the propagation of pressure waves, the position of the person to be measured is an important factor, since pulse transit times are strongly dependent on the vertical placement of sensors, due to gravitation. Therefore, PTT responses between two measurements are comparable only if the position of the person is exactly the same in both measurements. However, these time differences are smaller for a person in a supine position. Sensors of the type discussed here are aimed mainly for use during MRI measurements, where the patient is practically always in a supine position. This makes a comparison to PTT values more reasonable.

The obtained results can be utilized to determine such parameters as pulse wave velocity, blood oxygen saturation, and blood pressure in different parts of the human body. Furthermore, due to MR compatibility, these results, when combined and analyzed together with fMRI signals, offer new possibilities for the study of correlations between signals generated by neural activity and signals from various parts of the body.

\section{Acknowledgments}

The authors would like to thank M.Sc. (Tech.) students Tomasz A. Szczesńy and Adam Krysinski for their assistance. The study was partly supported financially by the SalWe Research Program for Mind and Body (Tekes-the Finnish Funding Agency for Technology and Innovation, Grant no. 1104/10) and Instrumentariumin Tiedesäätiö.

\section{References}

[1] J. F. Schenck, "The role of magnetic susceptibility in magnetic resonance imaging: MRI magnetic compatibility of the first and second kinds," Medical Physics, vol. 23, no. 6, pp. 815-850, 1996.

[2] J. Virtanen, Enhancing the compatibility of surgical robots with magnetic resonance imaging [Ph.D. thesis], Department of Mechanical Engineering, University of Finland, 2006.

[3] W. Shi, K. Shen, J. K. J. Li, G. H. Sigel, and R. Mezrich, "Fiber optic sensors for biomedical measurements in magnetic 
resonance imaging (MRI)," in Proceedings of the 39th Electronic Components Conference, pp. 479-481, May 1989.

[4] S. C. Chung, J. H. Kwon, B. Lee, J. H. Yi, H. J. Kim, and G. R. Tack, "Development of a magnetic-resonance-compatible photoplethysmograph amplifier for behavioral and emotional studies," Behavior Research Methods, vol. 40, no. 1, pp. 342 346, 2008.

[5] S. C. Chung, M. H. Choi, S. J. Lee et al., "Simultaneous measurement of PPG and functional MRI," in Proceedings of the 13th International Conference on Biomedical Engineering, vol. 23, pp. 161-164, 2009.

[6] J. Popp, V. V. . Tuchin, A. Chiou, and S. H. . Heinemann, Eds., Handbook of Biophotonics, vol. 2, Wiley, 2012.

[7] S. Del Bianco, F. Martelli, and G. Zaccanti, "Penetration depth of light re-emitted by a diffusive medium: theoretical and experimental investigation," Physics in Medicine and Biology, vol. 47, no. 23, pp. 4131-4144, 2002.

[8] T. Valery, Tissue Optics-Light Scattering Methods and Instruments for Medical Diagnosis, SPIE, 1st edition, 2000.

[9] F. D. Dagan, Biomedical Information Technology, Elsevier, Academic Press, 2008.

[10] H. S. S. Sorvoja, T. S. Myllylä, M. Y. Kirillin et al., "Noninvasive, MRI-compatible fibreoptic device for functional near-IR reflectometry of human brain," Quantum Electronics, vol. 40, no. 12, pp. 1067-1073, 2010.

[11] H. Obrig, M. Neufang, R. Wenzel et al., "Spontaneous low frequency oscillations of cerebral hemodynamics and metabolism in human adults," NeuroImage, vol. 12, no. 6, pp. 623-639, 2000.

[12] M. Cope, The application of near-infrared spectroscopy to noninvasive monitoring of cerebral oxygenation in the newborn infant [Ph.D. thesis], Department of Medical Physics and Bioengineering, University College London, 1991.

[13] T. S. Myllylä, A. A. Elseoud, H. S. S. Sorvoja et al., "Fibre optic sensor for non-invasive monitoring of blood pressure during MRI scanning," Journal of Biophotonics, vol. 4, no. 1-2, pp. 98107, 2011.

[14] Y. Tong and B. D. Frederick, “Time lag dependent multimodal processing of concurrent fMRI and near-infrared spectroscopy (NIRS) data suggests a global circulatory origin for lowfrequency oscillation signals in human brain," NeuroImage, vol. 53, no. 2, pp. 553-564, 2010.

[15] B. P. M. Imholz, W. Wieling, G. A. Van Montfrans, and K. H. Wesseling, "Fifteen years experience with finger arterial pressure monitoring: assessment of the technology," Cardiovascular Research, vol. 38, no. 3, pp. 605-616, 1998.

[16] D. B. McCombie, A. T. Reisner, and H. H. Asada, "Motion based adaptive calibration of pulse transit time measurements to arterial blood pressure for an autonomous, wearable blood pressure monitor," in Proceedings of the 30th Annual International Conference of the IEEE Engineering in Medicine and Biology Society (EMBS '08), pp. 989-992, August 2008.

[17] J. Sola, S. F. . Rimoldi, and Y. Allemann, "Ambulatory monitoring of the cardiovascular system: the role of pulse wave velocity," in New Developments in Biomedical Engineering, D. Campolo, Ed., 2010. 

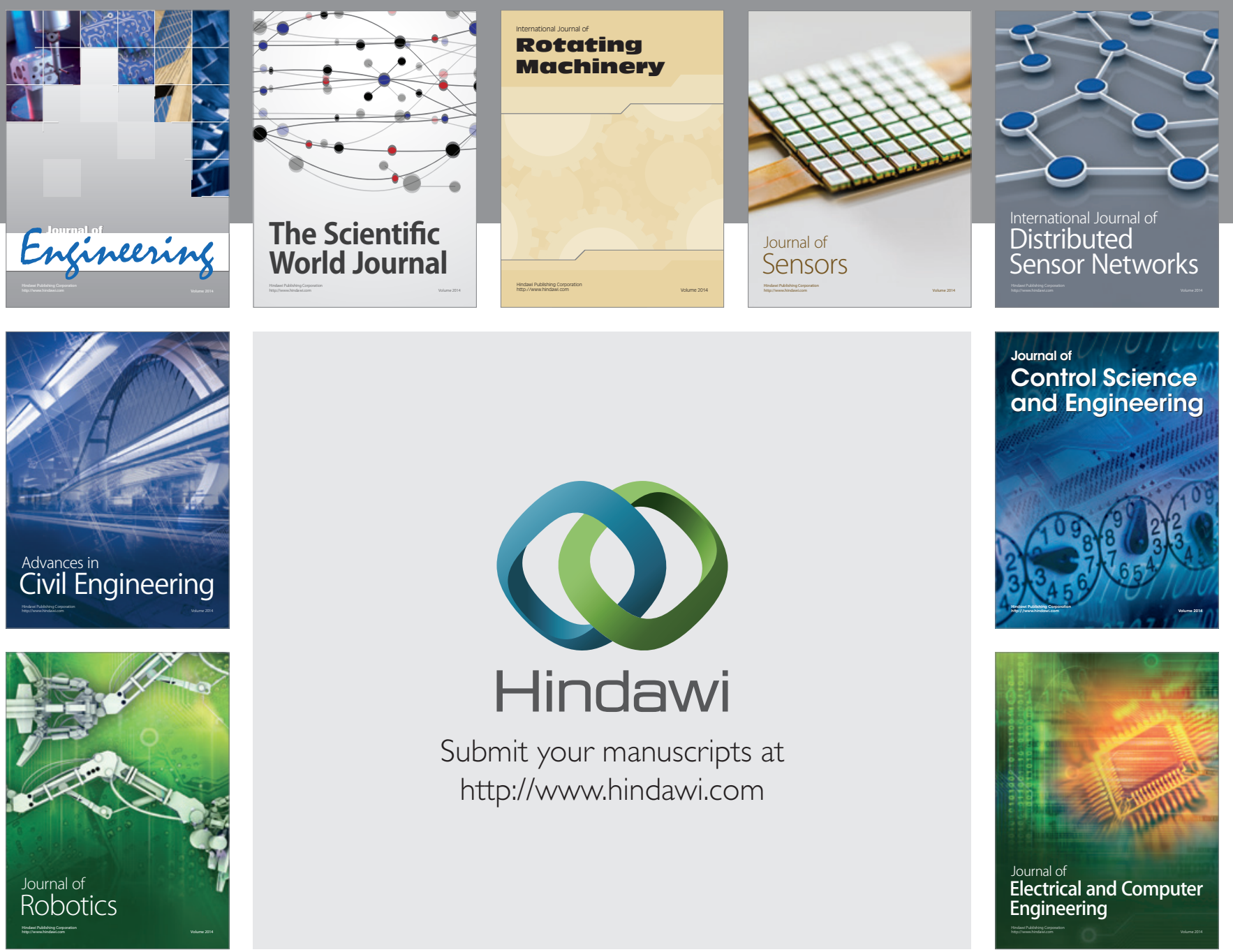

Submit your manuscripts at

http://www.hindawi.com
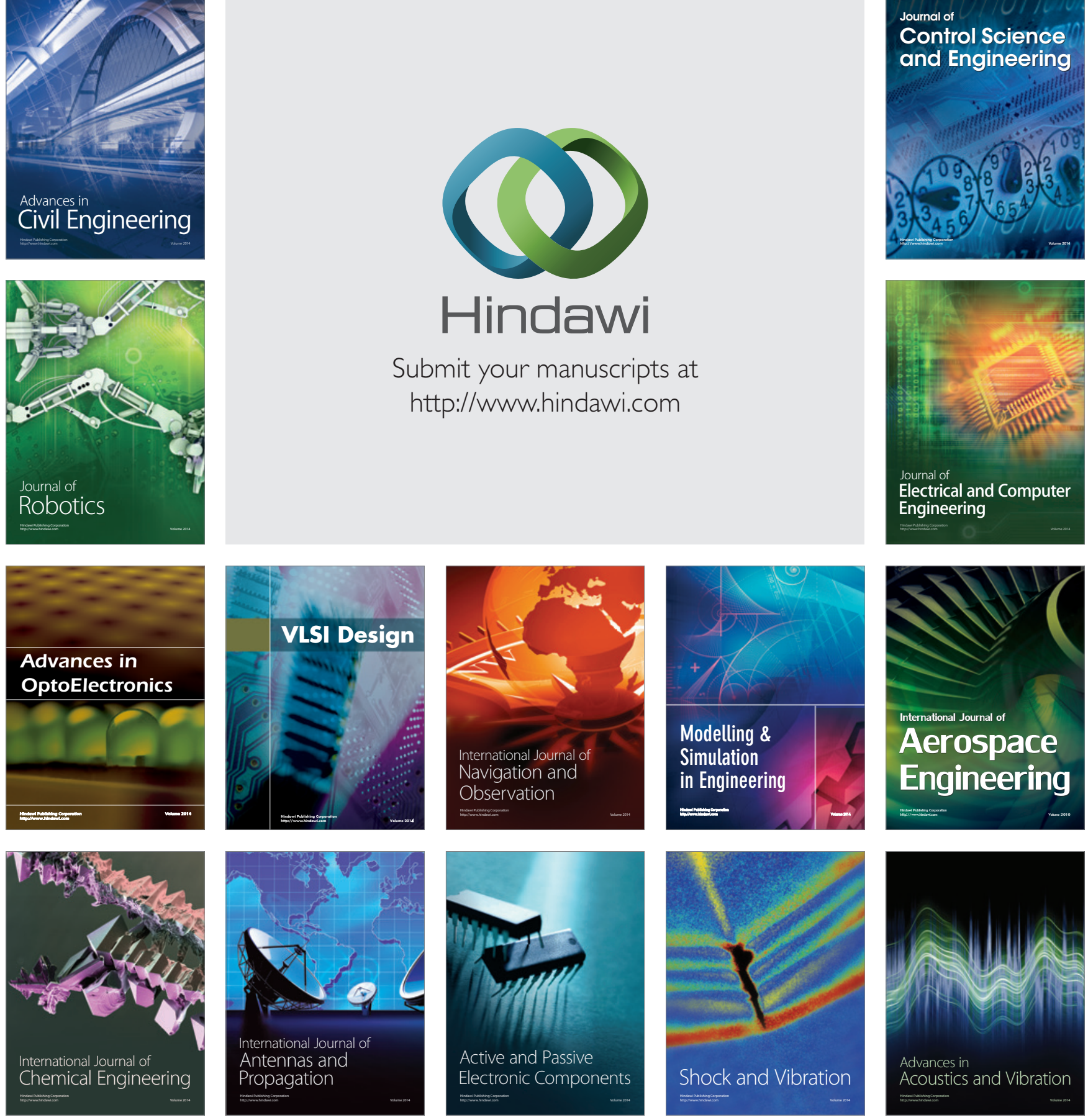